Journal of Engineering and Applied Sciences 14 (Special Issue 6): 9405-9413, 2019

ISSN: 1816-949X

(C) Medwell Journals, 2019

\title{
Study and Comparison of Channel Simulation Models for 5 G Applications
}

\author{
${ }^{1}$ Adheed H. Saloom, ${ }^{2}$ Hayam Alyasiri and ${ }^{1}$ Najim Abdallah \\ ${ }^{1}$ Department of Electrical Engineering, University of Al-Mustansiriya, Baghdad, Iraq \\ ${ }^{2}$ Ministry of Communication, Baghdad, Iraq \\ adalameed@yahoo.com
}

\begin{abstract}
One of the major problems associated with the development of the fifth generation in telecommunications systems is the development of radio propagation models operating in $\mathrm{mm}$ waves (the frequency spectrum beyond $6 \mathrm{GHz}$ ). In this study, different types of channels were studied and evaluated at different values of frequencies. The 3rd Generation Partnership Project (3GPP) TR 38.900 (Release 14) path loss model and Close-In (CI) path loss model, Stanford University Interim (SUI) path loss model are studied and compared both LOS and NLOS environment are investigated to analyze. These results were based on large-scale results and beam tracking across multiple frequencies ranging from $0-100 \mathrm{GHz}$. This document describes the different channel models including) typical microcell and macrocell propagation scenarios for urban areas) a basic model for integrating path loss, shadow fading for the typical scenarios. This research demonstrates that the 3GPP Model is accurate and stable for the NLOS state and it is independent of the frequencies beyond the first meter of propagation to the LOS environment. SUI Model found to be more accurate and relevant to the LOS environment, followed it CI Model.
\end{abstract}

Key words: $5 \mathrm{G}$ channel model, UMI, UMA, outdoor, indoor, millimeterwave

\section{INTRODUCTION}

The rapid growth of personal communication devices such as smart phones and tablets and the growing demand for data everywhere have prompted telecommunications networks to work on providing data with higher quality. Innovative technologies and new frequency bands such as millimeter waves are needed to meet this demand, leading to the development of the so-called 5th-Generation wireless communications (5G) (Tikhomirov et al., 2018).

The emerging $5 \mathrm{G}$ communications systems are expected to introduce revolutionary technologies with the use of new concepts and new possible architectural styles (Rappaport et al., 2013). It is therefore, important to develop new channel standards and models to assist engineers in designing the system. The channel characterization was done on the wavelength frequencies by two previous researchers.

In downtown Denver, non-line-of-sight broadband channels were examined at 9.6, 28.8 and $57.6 \mathrm{GHz}$ by Haneda et al. (2016). Samsung has been dynamic in measuring and modeling millimeter channels for modern mobile communications (Rappaport et al., 2017). In the band $81-86 \mathrm{GHz}$ Aalto conducted measurements of point-to-point communication on a road in Helsinki, Finland (Roh et al., 2014). In 28, 38 and $73 \mathrm{GHz}$, broadband measurements were carried out in UMI, UMA and/or internal scenarios (Rappaport et al., 2015a, b). At 28 and $73 \mathrm{GHz}$, spatial and temporal measurements were obtained in conjunction with beam tracing the omnidirectional path loss models were explored in dense urban situations (Thomas et al., 2016).

There are many current and ongoing campaign endeavors around the world focusing on the $5 \mathrm{G}$ measurement. They include METIS 2020 (Anonymous, 2015), COST 2100/COST (Anonymous, 2015), IC 1004 (Rappaport et al., 2013), ETSI mm wave (Rappaport et al., 2015a, b), NIST 5G mm wave Channel Model Alliance (Sun and Rappaport, 2017), MiWEBA (Maccartney et al., 2015), mmMAGIC (Sun et al., 2016) and NYU Wireless (Sakaguchi et al., 2015). METIS 2020, extensive studies across a wide range of frequency bands (up to $86 \mathrm{GHz}$ ) and a very large bandwidth $(100 \mathrm{MHz})$ contributed extensive studies in channel modeling, 3D polarization modeling, spherical and high spatial resolution. METIS channels consist of a map-based model, a random model and a hybrid model. The COST 2100 channel model can be an engineering-based randomized Channel Model (GSCM) where it can reproduce the random characteristics of

Corresponding Author: Adheed H. Saloom, Department of Electrical Engineering, University of Al-Mustansiriya, Baghdad, Iraq, adalameed@yahoo.com 
Multiple Input Output (MIMO) channels over time, frequency and space. The (New York University) NYU Wireless conducted and distributed large-scale urban propagation measurements at $28,38,60$ and $73 \mathrm{GHz}$ for both external and internal channels and provided large-scale and small-scale channel models (Rappaport et al., 2013).

This study presents the path loss models 3GPP TR 38.900 (Maccartney et al., 2015), Close In (CI) free space reference distance (Sun et al., 2016) and Stanford University Interim (SUI) (Sakaguchi et al., 2015). Using some sets of measurement data or monitoring of $\mathrm{mm}$ wave frequencies contributed by New York University (NYU), Nokia, University of Alburg (AAU), Qualcomm and Altoff University, the parameters in these three models were compared in both the UMI and UMA.

5G system requirements: The standard $5 \mathrm{G}$ mobile communication is also referred to as IMT-2020, aimed at improving the parameters of previous generation systems including the latest modification of the fourth generation or IMT-Advanced (Anonymous, 2016). The basic parameters of the $5 \mathrm{G}$ mobile communication system can be divided into three parts (Anonymous, 2016):

- enhanced Mobile BroadBand (eMBB) used for broadband internet access for resource intensive applications

- massive Machine Type Communications (mMTC) used for large-scale applications for internet of things

- Ultra-Reliable and Low Latency Communications (URLLC) for applications such as self-control vehicles

The $5 \mathrm{G}$ requirements are superior to $4 \mathrm{G}$ system parameters. Based on the applications and requirements of each sector with different priorities, so, the requirements in Table 1 do not necessarily represent the exact maximum values of fifth generation networks in general. For mMTC, the highest priority is communication density while spectrum efficiency and peak data rate are not important. For URLLCs, the highest priority is mobility and delay parameters for $\mathrm{eMBB}$, area traffic capacity user

Table 1: The fourth and fifth generation features

\begin{tabular}{lcc}
\hline Requirements & 4G (IMT-Advance) & 5G (IMT-2020) \\
\hline Area traffic capacity $\left(\mathrm{Mbps} / \mathrm{m}^{2}\right)$ & 0.1 & 10 \\
Peak data rate $(\mathrm{Gpbs})$ & 1 & 20 \\
User experienced data rate $(\mathrm{Mbps})$ & 10 & 100 \\
Spectrum efficiency (bps/Hz) & $\times 1$ & $\times 3$ \\
Mobility (km/h) & 350 & 500 \\
Latency (msec) & 10 & 1 \\
Connection density (dev/km) & $10^{5}$ & $10^{6}$ \\
\hline
\end{tabular}

data rate, mobility and peak data rate, although, communication density and latency are also important. So, the eMBB chip has the highest requirements and the most difficult to develop. General $5 \mathrm{G}$ prerequisites decide system parameters such as parameters of the physical layer including frequency band and bandwidth.

\section{G system parameters}

Communication range: To know the cell range of the fifth-generation mobile phone network, the BS antenna connection parameters should be used. By Thomas et al. (2016) it is proposed to use three antennas with the main beam of $120^{\circ}$. Therefore, if one antenna efficiently transmits the maximum spectrum, the cell range can reach a value of $44 \mathrm{~m}$. Given that the amplitude of the area motion should not necessarily have the maximum value at each point and that the close closure of Base Stations (BS) adds significantly to the cost of telecommunications services, it can be suggested that the cell range for eMBB can be greater. Furthermore, the draft ITU-R Report of October 2017 on IMT-2020 proposes different scenarios for $5 \mathrm{G}$ mobile networks in the eMBB section (Thomas et al., 2016). A desktop scenario or a high-end shopping center for User Equipment (UE) or an internal hotspot (eMBB). Can be fixed or moving slowly (speed does not exceed $10 \mathrm{~km} / \mathrm{h}$ ).

Data rates must reach maximum values suggesting that BS should be placed close to each other a distance of $20 \mathrm{~m}$. Dense Urban-eMBB. This scenario for an urban area where users do not move faster than $30 \mathrm{~km} / \mathrm{h}$ as the network structure can be divided into BS level at the macro level and and micro-level for access points. Rural-eMBB. Under this situation, the rural environment at a user speed of up to $500 \mathrm{~km} / \mathrm{h}$ this value corresponds to high-speed rail traffic. For $5 \mathrm{G}$ systems, many design objectives can be distinguished:

- Capacity of downlink enhancement up to $20 \mathrm{Gbps}$ latency reduction to $1 \mathrm{msec}$

- Mobility of user increases to $500 \mathrm{~km} / \mathrm{h}$

- $1 \mathrm{~m}$ devices per $\mathrm{km}^{2}$ can be connected to the network

Propagation of radio-wave: Radio frequency propagation effects vary according to signal frequency as shown Maccartney et al. (2015) UHF (300 MHz-3 the UHF (includes the bands $790-794 \mathrm{MHz}$ and $2.5 \mathrm{GHz}$. It is called a decimetric waves. Used for location, television, cell phone, relay, mobile and fixed communications. The decimeter radio waves propagate basically flat and suffer from reflections of smooth obstacles that meet the Rayleigh standard. SHF (3-30 GHz) includes the bands 3.4-3.8, 5.9, 25.5-27 GHz. These waves are commonly called 
centimeter waves and are mostly used in sequential and satellite communications. SHF signals indicate absorption of an experiment in air and air gases (mainly in oxygen and water vapor). SHF waves are widely used in mobile communications.

EHF $(30-300 \mathrm{GHz})$, called millimeter waves and are afterward presented into radio communications. The millimeter waves are propagated by geometric optics and are characterized by a refraction within the ambiguity of the environment and critical absorption in atmospheric dust and gases. However, the most advantage of $\mathrm{mmW}$ waves is the ability to assign broad frequency bands that give target values for a radio link capacitance, rather than specifying that the $\mathrm{EHF}$ radio spectrum does not operate intensively. In this study, path loss channels simulation are tested under three values of the carrier frequency (700 $\mathrm{MHz})$ in $\mathrm{UHF}$ bands $(3.6 \mathrm{GHz})$ in $\mathrm{SHF}$ bands and $(26 \mathrm{GHz})$ in $\mathrm{EHF}$ bands. These values are widely used by many researchs.

\section{Channel simulation models}

Line-of-sight probability: The status of the LOS is determined through a map-based approach, for example, through the placement of transmitters Access Point (AP) and receivers (UE) User devices and whether any buildings or walls prevent the direct path between AP and UE. The effects of objects not represented on the map such as trees, cars, furniture, etc. are calculated independently by using shading/blocking terms. The first model is the 3D 3GPP channel model in the UMA high-end User Equipment (UE) scenario for a height of $1.5 \mathrm{~m}$ (Anonymous, 2016):

$$
P(d)=\min \left(\frac{18}{d}, 1\right)\left(1-e^{-d / 63}\right)+e^{-d / 63}
$$

where, $d$ is the distance in $\mathrm{m}$. Second model is the $d_{1} / d_{2}$ model:

$$
P(d)=\min \left(\frac{d_{i}}{d}-1\right)\left(1-e^{-d / d_{2}}\right)+e^{-d / d_{2}}
$$

where, $d_{1}$ and $d_{2}$ are parameters are estimated to optimized $\mathrm{P}(\mathrm{d})$ : New York University (NYU) suggested the third model is which is basically a $3 \mathrm{G} \mathrm{PP}$ presentation of $\mathrm{d}_{1} / \mathrm{d}_{2}$ but with a square term for the LOS probability.

$$
P(d)=\left(\min \left(\frac{d_{i}}{d}-1\right)\left(1-e^{-d / d_{2}}\right)+e^{-d / d_{2}}\right)^{2}
$$

Table 2 and 3 summarizes the results and compares the three models with the data in Fig. 1. In terms of the average Square Error (MSE) between the LOS probability
Table 2: Cell types parameters of cell types based on their bandwidth (Anonymous, 2015)

\begin{tabular}{llll}
\hline Cell types & $\mathrm{Pt}(\mathrm{W})$ & Cell range $(\mathrm{km})$ & Applications \\
\hline Mac-cell & $10-50$ & $8-30$ & Outdoor \\
Mic-cell & $1-10$ & $0.2-2$ & Outdoor/indoor \\
Pico-cell & $0.25-1$ & $0.1-0.2$ & Outdoor $/$ indoor \\
Femto-cell & $0.001-0.25$ & $0.01-0.1$ & Indoor \\
\hline
\end{tabular}

Table 3: Comparison of the three LOS probability models for UMA situation

\begin{tabular}{llrc}
\hline Types of models & $\mathrm{d}_{1}$ & $\mathrm{~d}_{2}$ & Mean Square Error (MSE) \\
\hline 3GPP Model & 19 & 62 & 0.0210 \\
$\mathrm{~d}_{1} / \mathrm{d}_{2}$ Model & 21 & 65 & 0.0171 \\
NYU Model & 21 & 161 & 0.0140 \\
\hline
\end{tabular}

Table 4: Comparison of the three LOS probability models for UMI situation \begin{tabular}{llll}
\hline Types of model & $\mathrm{d}_{1}$ & $\mathrm{~d}_{2}$ & Mean Square Error (MSE) \\
\hline
\end{tabular} 3 GPP UMA $\mathrm{d}_{1} / \mathrm{d}_{2}$ Model NYU (squared)

\begin{tabular}{rrc}
$\mathrm{d}_{1}$ & $\mathrm{~d}_{2}$ & Mean Square Error (MSE) \\
19 & 62 & 0.0204 \\
21 & 65 & 0.0135 \\
21 & 161 & 0.0103 \\
\hline
\end{tabular}

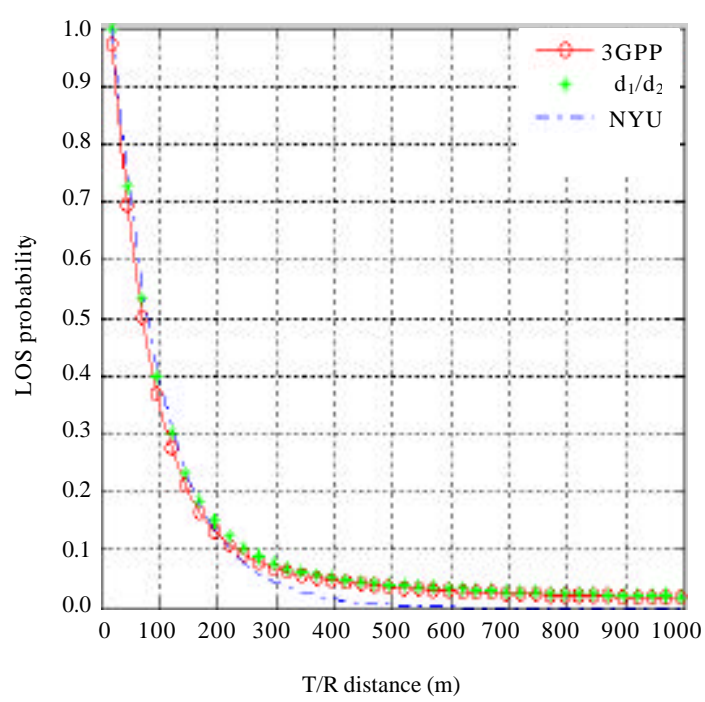

Fig. 1: UMA LOS probability for the three models considered

of data and models, the NYU Model was lower than the MSE but the difference was small. Given that the current 3GPP UMA Model conforms to reasonable data and includes 3D Mode support for UEs, it is recommended to use the current $3 \mathrm{GPP}$ LOS Model for UMA for frequencies above $6.0 \mathrm{GHz}$. For the UMI scenario, Table 4 summarizes the comparison of the three models with the data in Fig. 2. In terms of average Square Error (MSE) between the LOS probability of data and models, the NYU (square) model was the lowest MSE but the distinction was small.

Path loss models

Closed-In (CI) path loss model: The equation for the CI Model is givin by Eq. (4) (Anonymous, 2016): 


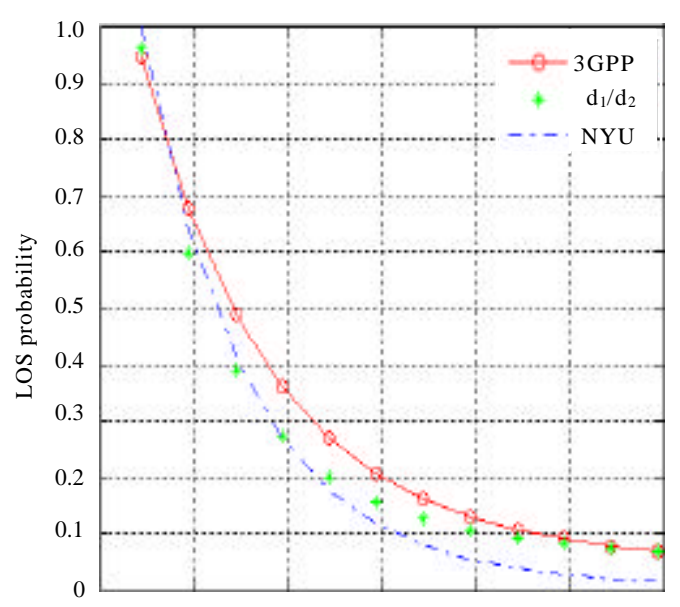

$\mathrm{T} / \mathrm{R}$ distance $(\mathrm{m})$

Fig. 2: UMI LOS probability for the three models considered

$$
\mathrm{PL}^{\mathrm{Cl}}(\mathrm{f}, \mathrm{d})=\mathrm{FSPL}(\mathrm{f}, 1)[\mathrm{dB}]+10 \mathrm{n} \log \left(\frac{\mathrm{d}}{1 \mathrm{~m}}\right)
$$

Where:

$$
\begin{array}{lll}
\mathrm{PL}^{\mathrm{CI}}(\mathrm{f}, \mathrm{d}) & =\text { The mean Path Loss } \\
\mathrm{n} & =\text { The Path Loss Exponent (PLE) } \\
\mathrm{d} & =\begin{array}{l}
\text { The separation distance between both } \\
\text { transmitter and receiver }
\end{array}
\end{array}
$$

FSPL (f, $1 \mathrm{~m})=$ The Free Space path loss in $\mathrm{dB}$ at a T-R separation distance of $1 \mathrm{~m}$ at the carrier frequency $f$ by Eq. 5

$$
\operatorname{FSPL}(\mathrm{F}, 1 \mathrm{~m})=20 \log _{10}\left(\frac{4 \pi \mathrm{f}}{\mathrm{c}}\right)
$$

where, $\mathrm{c}$ is the speed of light. The CI Model is based on the basic principles of wireless propagation and dating to Friis and Bullington, PLE where the value is 2 in the free space as shown by Friis and the value 4 for the model of the terrestrial $\mathrm{X}$-ray diffraction propagation model. Use $\mathrm{d}_{0}=1 \mathrm{~m}$ in mmWave path loss models because base stations will be shorter or mounted indoors and closer to obstacles (Anonymous, 2016).

3GPP path loss model: The 3GPP path loss model UMA LOS consists of two sections separated by a breakpoint distance where the attenuation attenuation increases beyond the cutoff distance as shown in Table 5-7 (MacCartney and Rappaport, 2017):

$$
\mathrm{PL}_{\text {UMALOS }}=\left\{\begin{array}{c}
\mathrm{PL}_{1}=32.4+20 \log _{10}\left(\mathrm{~d}_{3 \mathrm{D}}\right)+20 \log _{10}(\mathrm{fc}) \\
10 \mathrm{~m} \leq \mathrm{d}_{2 \mathrm{D}} \leq \mathrm{d}_{\mathrm{BP}} \\
\mathrm{PL}_{2}=32.4+20 \log _{10}\left(\mathrm{~d}_{3 \mathrm{D}}\right)+20 \log _{10}(\mathrm{fc})-10 \log _{10} \\
\left(\left(\mathrm{~d}_{\mathrm{BP}}\right)^{2}+\left(\mathrm{h}_{\mathrm{BS}}-\mathrm{H}_{\mathrm{UT}}\right)^{2}\right), \mathrm{d}_{\mathrm{BP}} \leq \mathrm{d}_{2 \mathrm{D}} \leq 5 \mathrm{~km}
\end{array}\right.
$$

Table 5: Gives the values of CI parameters used in this research

\begin{tabular}{lcccc}
\hline Scenario/Enviornment & Models & Freq. (GHz) & Distance (m) & PLE (n) \\
UMI & & & & \\
LOS & CI & $0.7,3.6,26$ & $1-1000$ & 2.0 \\
NLOS & & & & 3.1 \\
UMA & & & & \\
LOS & CI & $0.7,3.6,26$ & $1-5000$ & 2.0 \\
NLOS & & & & 3.3 \\
\hline
\end{tabular}

Table 6: Gives the values of $3 \mathrm{GPP}$ parameters used in this research

\begin{tabular}{lll}
\hline Sce. & Models/Env. & Parameters \\
\hline UMI & 3GPP & \\
& LOS & $\mathrm{h}_{\mathrm{UT}}=1.5 \mathrm{~m}, \mathrm{~h}_{\mathrm{BS}}=10 \mathrm{~m}$ \\
& NLOS & \\
UMA & 3GPP & $\mathrm{h}_{\mathrm{UT}}=1.5 \mathrm{~m}, \mathrm{~h}_{\mathrm{BS}}=25 \mathrm{~m}$ \\
& LOS & \\
\hline
\end{tabular}

Table 7: Gives the values of SUI parameters used in this research

Sce./Env. Parameters

UMI

LOS

NLOS

UMA

LOS

NLOS

$$
\mathrm{d}_{3 \mathrm{D}}=\sqrt{\mathrm{d}_{2 \mathrm{D}}{ }^{2}+\mathrm{h}_{\mathrm{UT}}^{2}}
$$

Breakpoint distance $\mathrm{d}_{\mathrm{BP}}=4 \mathrm{hBShUTf} / \mathrm{c}$ where, $\mathrm{fc}$ is the middle frequency in $\mathrm{Hz}, \mathrm{c}=3.0 \times 108 \mathrm{~m} / \mathrm{sec}$ is the propagation velocity in free space and $h_{B S}$ and $h_{U T}$ are the compelling antenna heights at the $\mathrm{BS}$ and the UT, respectively. The UMA NLOS path loss model in 3GPP (Rappaport et al., 2015) is taken directly from ITU-R (MacCartney and Rappaport, 2017):

$$
\begin{gathered}
\mathrm{PL}_{\text {UMA-NLOS }}=\max \left(\mathrm{PL}_{\text {UMA-LOS }}, \mathrm{PL}_{\text {UMA-NLOS }}\right) \text { for } 10 \mathrm{~m} \leq \\
\mathrm{d}_{2 \mathrm{D}} \leq 5 \mathrm{~km}, \mathrm{PL}_{\text {UMA-NLOS }}=13.54+39.08 \log _{10}\left(\mathrm{~d}_{3 \mathrm{D}}\right)+ \\
20 \log _{10}(\mathrm{fc})-0.6\left(\mathrm{~h}_{\mathrm{UT}}-1.5\right)
\end{gathered}
$$

The UMI-LOS path loss model in 3GPP is:

$$
\begin{gathered}
\mathrm{PL}_{\mathrm{UMI-LOS}}=\left\{\begin{array}{l}
\mathrm{PL}_{1}=32.4+21 \log _{10}\left(\mathrm{~d}_{3 \mathrm{D}}\right)+20 \log _{10}(\mathrm{fc}) \\
10 \mathrm{~m} \leq \mathrm{d}_{2 \mathrm{D}} \leq \mathrm{d}_{\mathrm{BP}} \\
\mathrm{PL}_{2}=32.4+40 \log _{10}\left(\mathrm{~d}_{3 \mathrm{D}}\right)+20 \log _{10}\left(\mathrm{~d}_{\mathrm{BP}}\right)^{2}(8) \\
+\left(\mathrm{h}_{\mathrm{BS}}\right)-\mathrm{H}_{\mathrm{UT}}{ }^{2} \mathrm{~d}_{\mathrm{BP}} \leq \mathrm{d}_{2 \mathrm{D}} \leq 5 \mathrm{~km}
\end{array}\right. \\
\mathrm{d}_{3 \mathrm{D}}=\sqrt{\mathrm{d}_{2 \mathrm{D}}{ }^{2}+\mathrm{h}_{\mathrm{UT}}{ }^{2}}
\end{gathered}
$$




$$
\begin{gathered}
\mathrm{PL}_{\mathrm{UMI}-\mathrm{NLOS}}=\max \left(\mathrm{PL}_{\mathrm{UMA}-\mathrm{LOS}}, \mathrm{PL}_{\mathrm{UMA}-\mathrm{NLOS}}\right) \\
\text { for } 10 \mathrm{~m} \leq \mathrm{d}_{2 \mathrm{D}} \leq 5 \mathrm{~km}
\end{gathered}
$$

Stanford University Interim (SUI) Model: SUI path loss model for $\mathrm{UHF} / \mathrm{microwave}$ band from the SUI Model for $\mathrm{fc}$ $2 \mathrm{GHz}$ (Anonymous, 2017):

$$
\begin{gathered}
\mathrm{PL}_{\mathrm{SUI}}(\mathrm{d})=\mathrm{PL}\left(\mathrm{d}_{\mathrm{o}}\right)+10 \mathrm{n} \log 10\left(\frac{\mathrm{d}}{\mathrm{d}_{0}}\right)+\mathrm{X}_{\mathrm{fc}}+\mathrm{X}_{\mathrm{RX}}+\mathrm{X}_{\sigma} \\
\mathrm{PL}\left(\mathrm{d}_{0}\right)=20 \cdot \log _{10}\left(\frac{4 \pi \mathrm{d}_{0}}{\lambda}\right) \\
\mathrm{n}=\mathrm{a}-\mathrm{b} \cdot \mathrm{h}_{\mathrm{TX}}+\frac{\mathrm{c}}{\mathrm{h}_{\mathrm{TX}}} \\
\mathrm{X}_{\mathrm{fc}}=6 \cdot \log _{10}\left(\frac{\mathrm{f}_{\mathrm{MHz}}}{2000}\right) \cdot \mathrm{fc}>2 \mathrm{GHz} \\
\mathrm{X}_{\mathrm{RX}}=-10.8 \log _{10}\left(\frac{\mathrm{h}_{\mathrm{RX}}}{2}\right)
\end{gathered}
$$

where, - wavelength in meters, $\mathrm{PL}\left(\mathrm{d}_{0}\right)$ in Eq. 10 shows the loss of free space in $\mathrm{dB}$ at a close reference distance $\mathrm{d} 0 \mathrm{X}_{\mathrm{fc}}$ and $\mathrm{X}_{\mathrm{RX}}$ in Eq. 11 and 12 indicate the correction factors for the frequency and receiver heights, respectively and Eq. 10 is the typical random random shading variable with an average of $0 \mathrm{~dB}$ and the standard deviation $[\mathrm{dB}]$ such as $8.2<10.6 \mathrm{~dB}$. The $\mathrm{fMHz}$ in Eq. 11 is the frequency of the carrier (fc) in $\mathrm{MHz} \mathrm{h}_{\mathrm{TX}}$ and $\mathrm{h}_{\mathrm{RX}}$ indicate the height of the Transmitting antenna (TX) and the Receiver (RX) in meters, respectively. Parameters a-c in Eq. 12 are constants used to model the types of terrain encountered in the service area (Anonymous, 2017).

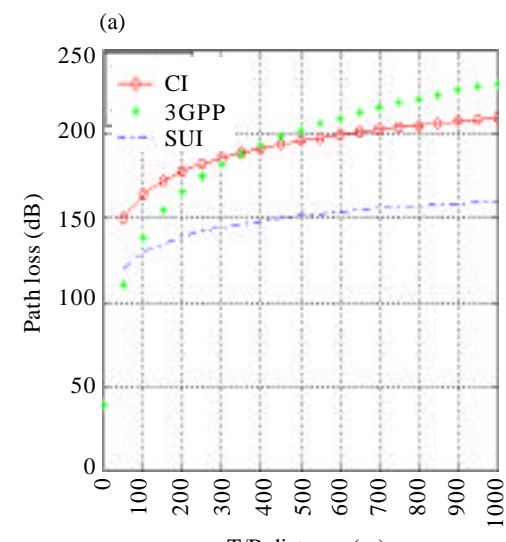

$\mathrm{T} / \mathrm{R}$ distance $(\mathrm{m})$

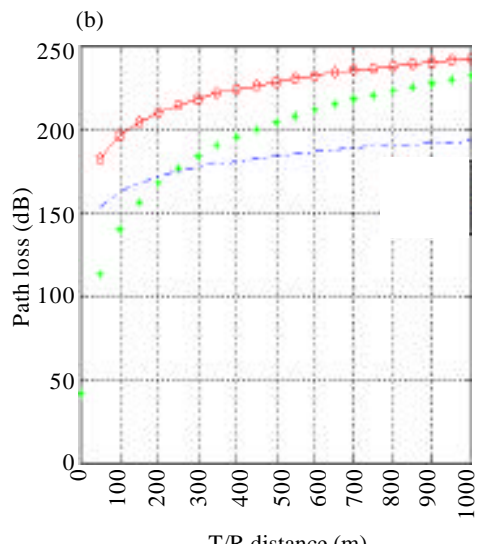

$\mathrm{T} / \mathrm{R}$ distance $(\mathrm{m})$
In order to modify the SUI path loss model using measurements of 3.6 and $26 \mathrm{GHz}$ NLOS, we simply find a slope correction factor such as Eq. 10 agree to the free-path loss model in free-reference space (Rappaport et al., 2013) which uses PLEs as shown in Table 7. In order to modify the SUI path loss model using measurements of 3.6 and $26 \mathrm{GHz} \mathrm{NLOS}$, we simply find a slope correction factor such as Eq. 10 agree with the free path loss model in free reference space (Rappaport et al., 2013) which uses PLEs as shown in Table 7 that the SUI Model contains more parameters that allow for frequency and elevation modulation. Thus, the SUI Model is more general and fully appropriate.

Modified SUI path loss model: The modified SUI Model for mmWave in NLOS environments (Anonymous, 2017):

$$
\mathrm{PL}_{\text {SUI, Mod }}[\mathrm{dB}](\mathrm{d})=\alpha \mathrm{NLOS} \times\left(\operatorname{PLSUI}(\mathrm{d})-\operatorname{PLSUI}\left(\mathrm{d}_{0}\right)\right)+\mathrm{PL}\left(\mathrm{d}_{0}\right)+\mathrm{X}_{\sigma}
$$

where, - NLOS is the mean slope correction factor (unitless) obtained directly from the NLOS mpirical results. For the LOS environment, the Friis FS path loss formula was used and the - LOS regression correction factor was found as follows:

$$
\mathrm{PL}_{\mathrm{FS}, \operatorname{Mod}}[\mathrm{dB}](\mathrm{d})=\alpha \operatorname{LOS} \times\left(\operatorname{PLFS}(\mathrm{d})-\operatorname{PLFS}\left(\mathrm{d}_{0}\right)\right)+\operatorname{PL}\left(\mathrm{d}_{0}\right)+\mathrm{X}_{\sigma}
$$

\section{RESULTS AND DISCUSSION}

Using the five large-scale propagation path loss models presented in section 5 and the outdoor measurement data at both $700 \mathrm{MHz}$ and 3.6 and $26 \mathrm{GHz}$, path loss parameters are analyzed and compared.

\section{Comparison between different models}

UMI-(LOS and NLOS): From Fig. 3, we note that the 3GPP Model is worst one because it is not affected by changing

(c)

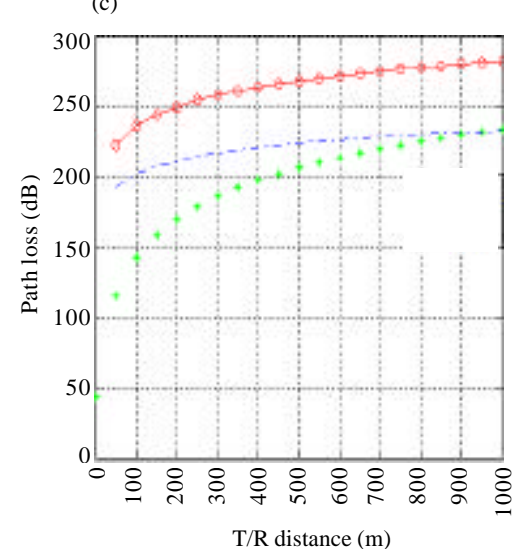

Fig. 3: UMI-LOS (CI, 3GPP and SUI) received power for carrier frequency models vs. Tx/Rx distance: a) $\mathrm{f}=700 \mathrm{MHz}$; ) $\mathrm{f}=3.6 \mathrm{GHz}$ and $\mathrm{c}) \mathrm{f}=26 \mathrm{GHz}$ 

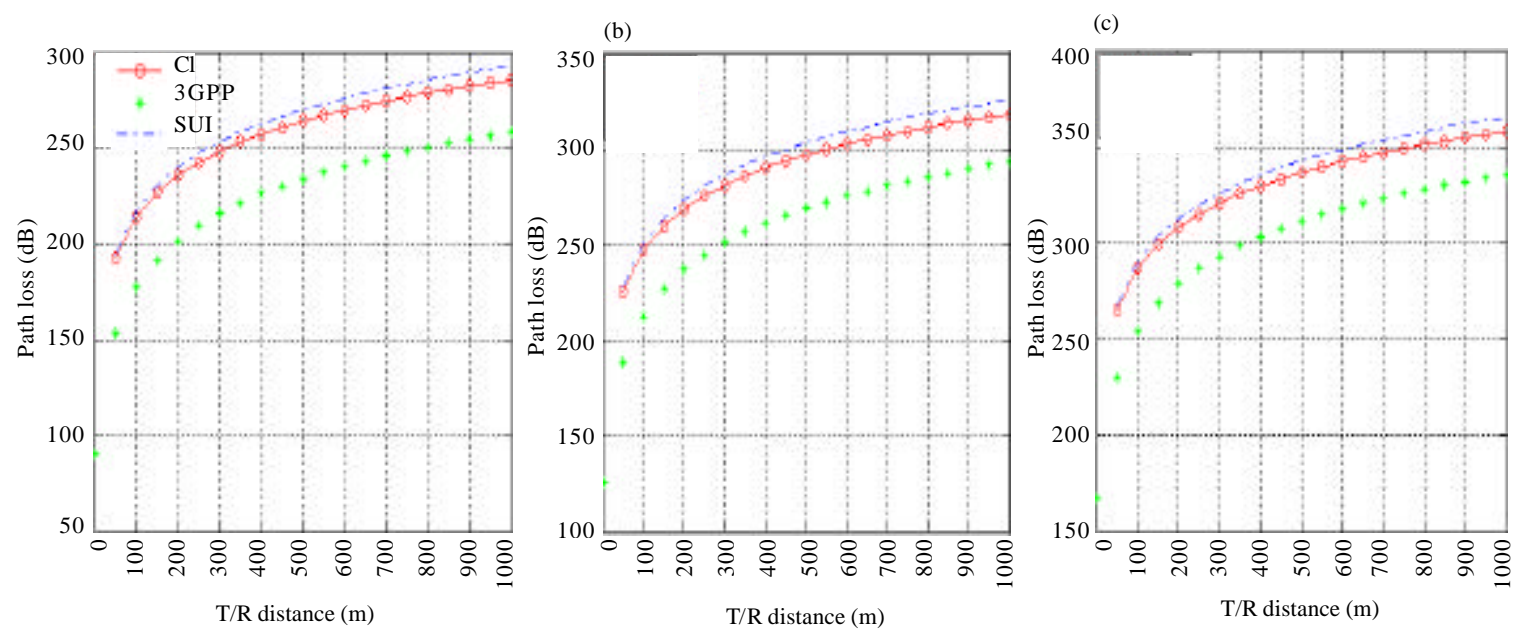

Fig. 4: UMI-LOS (CI, 3GPP and SUI) received power for carrier frequency models vs. Tx/Rx distance: a) $\mathrm{f}=700 \mathrm{MHz}$; b) $\mathrm{f}=3.6 \mathrm{GHz}$ and $\mathrm{c}) \mathrm{f}=26 \mathrm{GHz}$
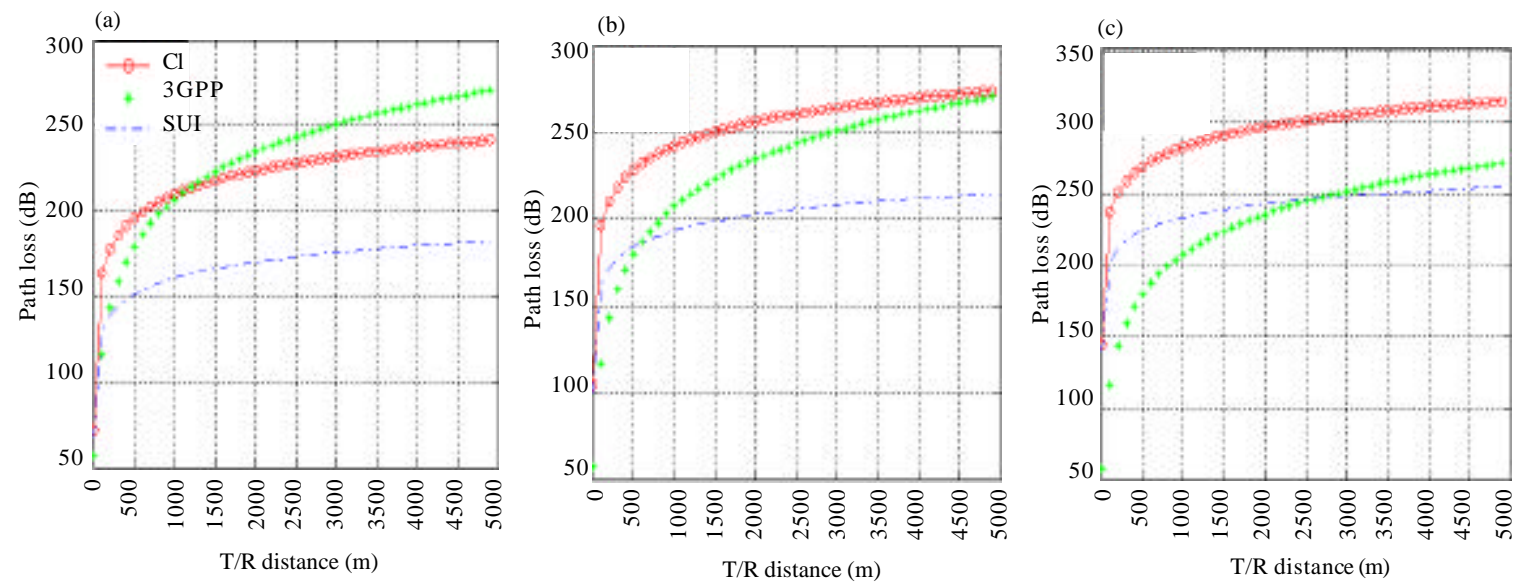

Fig. 5: UMI-LOS (CI, 3G PP and SUI) path loss for carrier frequency models vs. Tx/Rx distance: a) $\mathrm{f}=700 \mathrm{MHz}$; b) $\mathrm{f}=3.6 \mathrm{GHz}$ and c) $\mathrm{f}=26 \mathrm{GHz}$

the frequency range, therefore, we conclude SUI is the best one of the different values of frequency and then CI (Fig. 4). We note that the performance of $3 \mathrm{GPP}$ is the best one and then CI but SUI is the worst case for the three ranges of frequencies. Finally, for the case UMI scenario $3 \mathrm{GPP}$ unsuccessful for the case of LOS but in case of NLOS, it is best performed with respect of frequency.

UMA-(LOS and NLOS): From Fig. 5, we also note 3GPP Model is not affected by changing the frequency, therefore, SUI is the best path loss with respect to T/R distance for different frequency values. Then, $\mathrm{CI}$ on the other hand in Fig. 6, the 3GPP Model performance is the best one for the case of NLOS then CI and SUI is the last one (Fig. 6-10).

\section{Received power for different models}

UMI (LOS and NLOS): For LOS case (Fig. 7) the 3GPP Model is the worst one and SUI is the best then CI. but (Fig. 8) for the case of NLOS the $3 \mathrm{GPP}$ is best received power then $\mathrm{CI}$ and the last SUI.

UMA (LOS and NLOS): From Fig. 9, we also note 3GPP Model is not affected by changing the frequency, therefore, SUI is the best path loss with respect to $\mathrm{T} / \mathrm{R}$ distance for different frequency values. Then $\mathrm{CI}$ on the other hand in Fig. 10 the 3GPP Model performance is the best one for the case of NLOS then CI and SUI is the last one. 

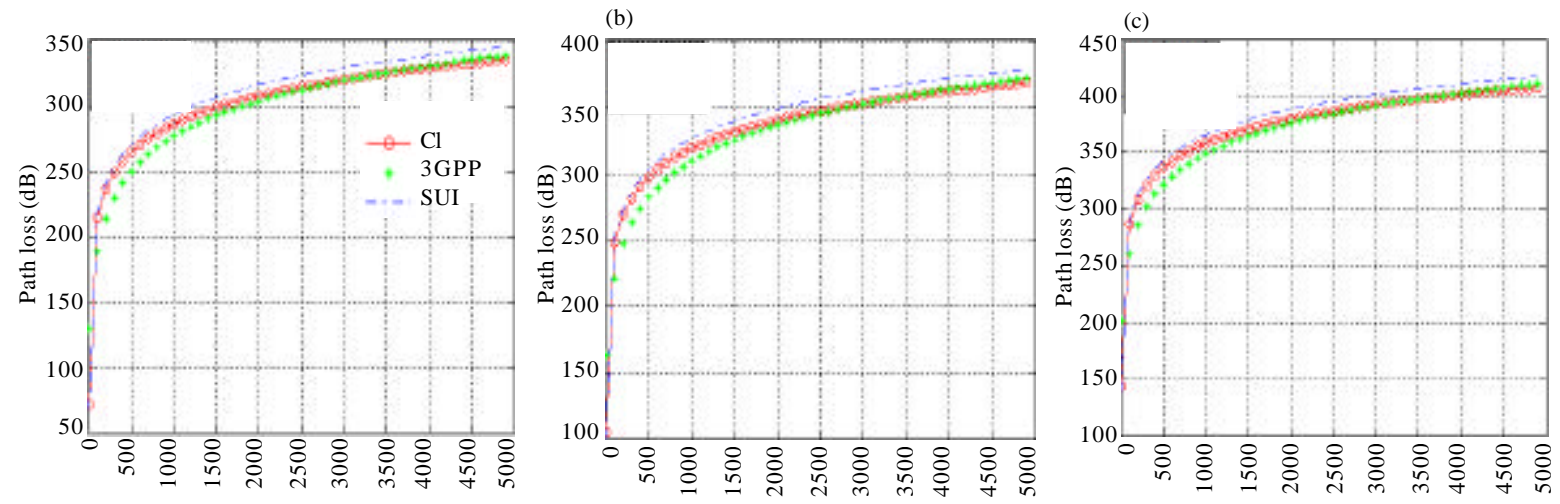

Fig. 6: UMI-NLOS (CI, 3GPP and SUI) received power for carrier frequency models vs. Tx/Rx distance: a) $\mathrm{f}=700 \mathrm{MHz}$; b) $\mathrm{f}=3.6 \mathrm{GHz}$ and c) $\mathrm{f}=26 \mathrm{GHz}$

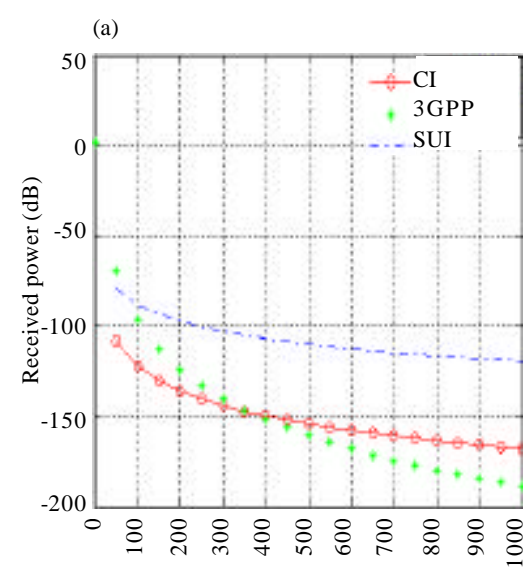

$\mathrm{T} / \mathrm{R}$ distance $(\mathrm{m})$

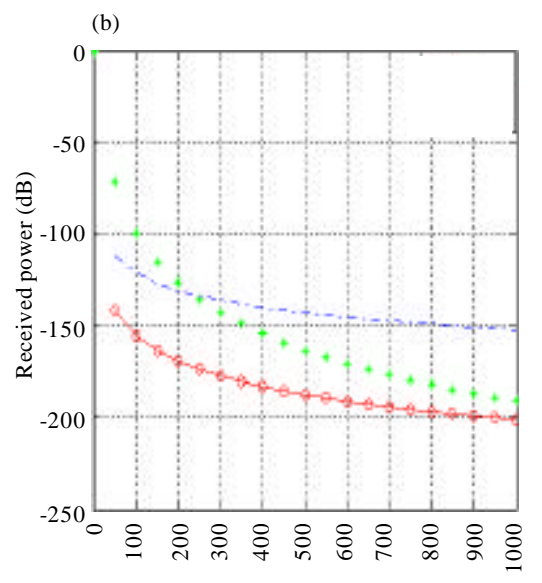

$\mathrm{T} / \mathrm{R}$ distance $(\mathrm{m})$

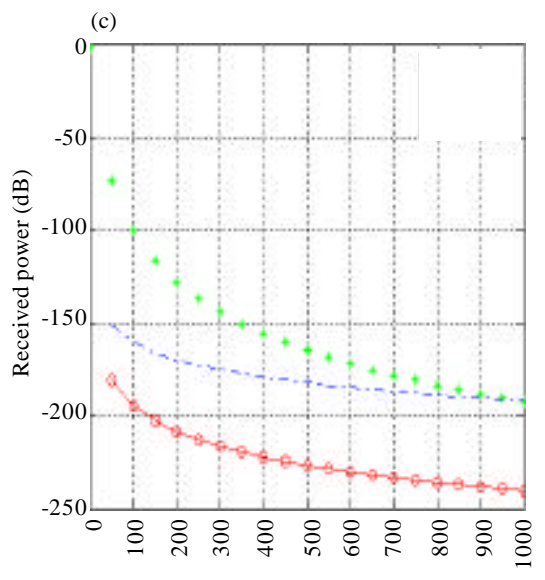

$\mathrm{T} / \mathrm{R}$ distance (m)

Fig. 7: UMI-LOS (CI, 3GPP and SUI) received power for carrier frequency models vs. Tx/Rx distance: a) $\mathrm{f}=700 \mathrm{MHz}$; b) $\mathrm{f}=3.6 \mathrm{GHz}$ and $\mathrm{c}) \mathrm{f}=26 \mathrm{GHz}$
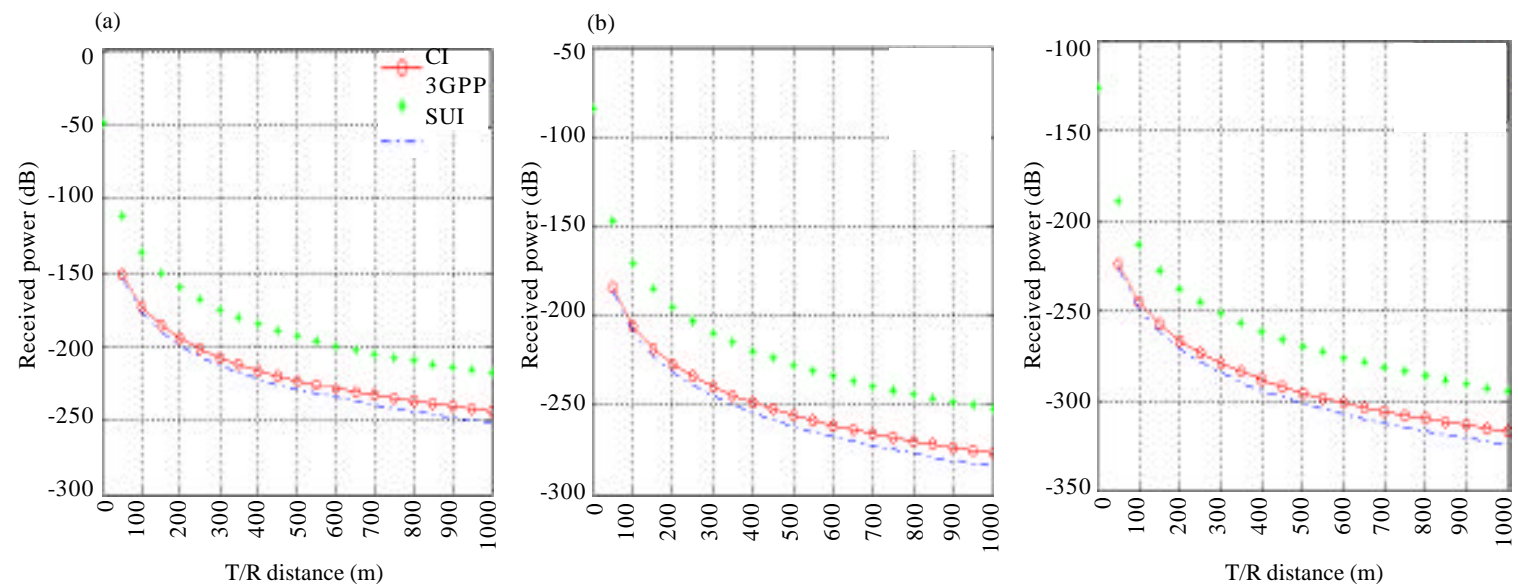

Fig. 8: UMI-NLOS (CI, 3GPP and SUI) received power for carrier frequency models vs. Tx/Rx distance: a) $\mathrm{f}=700 \mathrm{MHz}$; b) $\mathrm{f}=3.6 \mathrm{GHz}$ and c) $\mathrm{f}=26 \mathrm{GHz}$ 

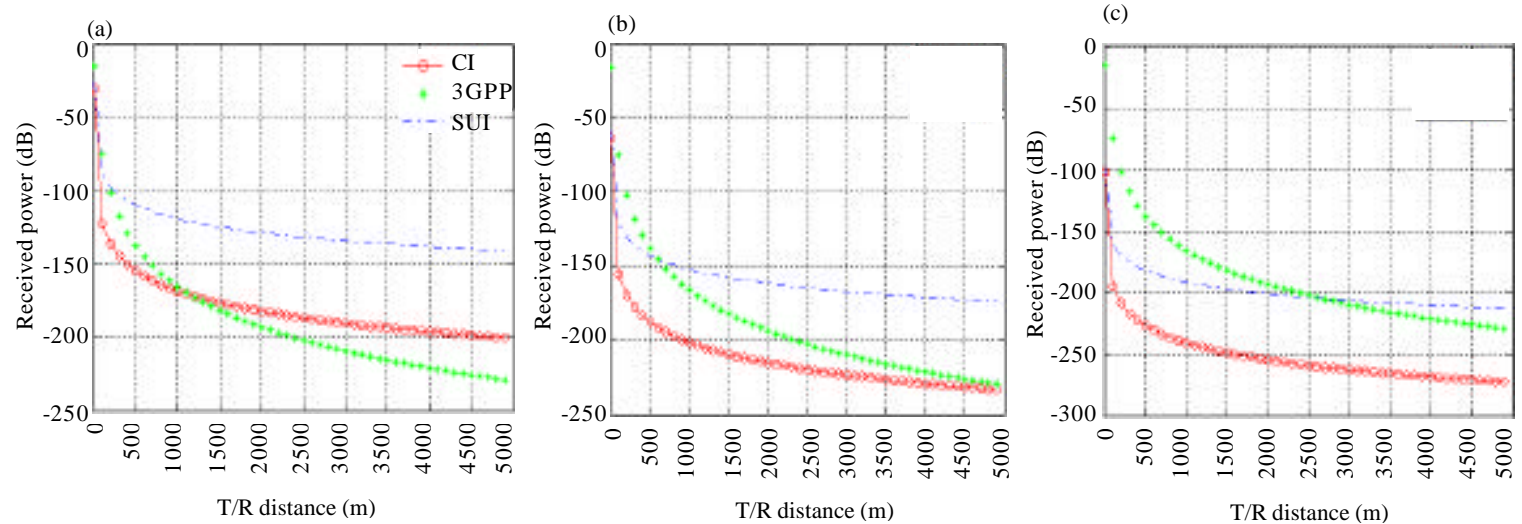

Fig. 9: UMA-LOS (CI, 3GPP and SUI) received power for carrier frequency models vs. Tx/Rx distance: a) $\mathrm{f}=700 \mathrm{MHz}$; b) $\mathrm{f}=3.6 \mathrm{GHz}$ and c) $\mathrm{f}=26 \mathrm{GHz}$

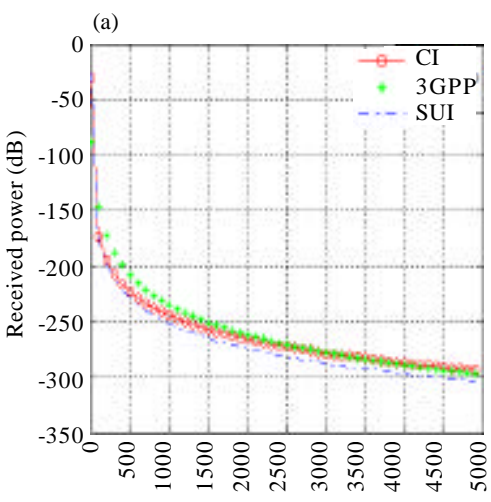

$\mathrm{T} / \mathrm{R}$ distance $(\mathrm{m})$

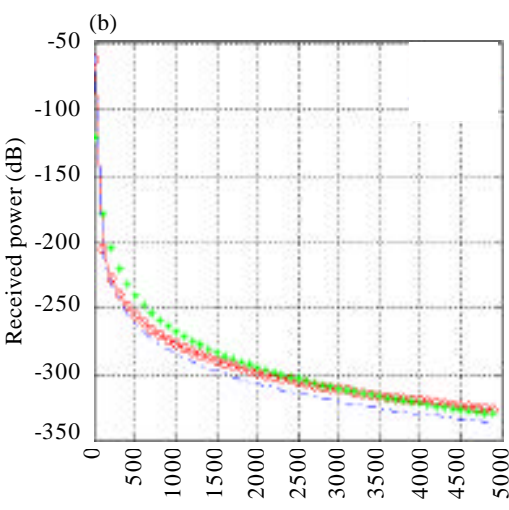

$\mathrm{T} / \mathrm{R}$ distance $(\mathrm{m})$

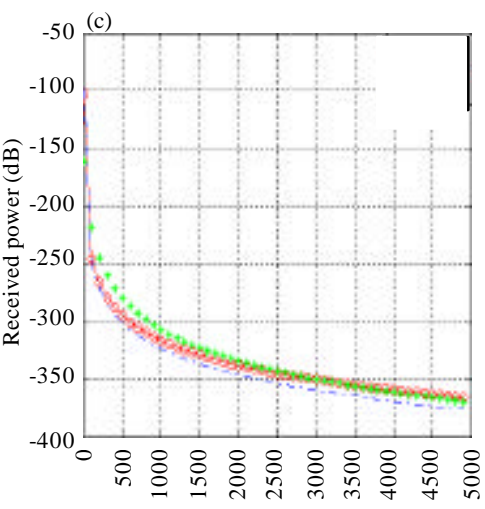

$\mathrm{T} / \mathrm{R}$ distance $(\mathrm{m})$

Fig. 10: UMA-NLoS (CI, 3GPP and SUT) path loss for carrier frequency models vs. Tx/Rx distance: a) $\mathrm{f}=700 \mathrm{MHz}$; b) $\mathrm{f}=3.6 \mathrm{GHz}$ and c) $\mathrm{f}=26 \mathrm{GHz}$

\section{CONCLUSION}

In this study, we given a comparison of the $3 \mathrm{GPP}, \mathrm{CI}$ and SUI path loss models within the mmWave frequency bands, utilizing measured data and ray-tracing from $0.5-100 \mathrm{GHz}$ gotten from diverse datasets from research groups over the world. The CI Model is physically tied to the transmitter power employing a close in free space reference and standardizes all measurements around an inherent $1 \mathrm{~m}$ free space reference distance that's physically based, hence, permitting simple utilize for changing distances without a calculator, through the utilize of just a single parameter (PLE or $n$ ).

An in-depth consider on the existing 3GPP (11) UMA/UMI LOS and NLOS path loss models for frequencies from $0.5-100 \mathrm{GHz}$ and found that no considerable experimental prove exists to date to bolster selection of this model by ITU-R. Given that few work existed within the literature and the questionable parameters utilized in $3 \mathrm{GPP}$ and ITU standards. The LOS SUI path loss, a great match with the CI LOS Model in (1) which indicated a SF of 4-6 dB. Additionally, the NLOS 3GPP Model, resulted in good path loss compared to the CI and SUI.

\section{REFERENCES}

Anonymous, 2015. 5G channel model for bands up to $100 \mathrm{GHz}$. Aalto University Executive Education, Helsinki, Finland.http://www.5gworkshops. com/5G_Channel_Model_for_bands_up_to100_G $\mathrm{Hz}(2015-12-6) \cdot \mathrm{pd} \bar{f}$

Anonymous, 2016. 3GPP TR 38.913: Scenarios and requirements for next generation access technologies. Green Plains Partners Company, Nebraska, USA.

Anonymous, 2017. 38.901: Study on channel model for frequencies from 0.5 to $100 \mathrm{GHz}$. Green Plains Partners Company, Nebraska, USA. 
Haneda, K., J. Zhang, L. Tan, G. Liu and Y. Zheng et al., 2016. 5G 3GPP-like channel models for outdoor urban microcellular and macrocellular environments. Proceedings of the 2016 IEEE 83rd International Conference on Vehicular Technology (VTC Spring), May 15-18, 2016, IEEE, Nanjing, China, ISBN:978-1-5090-1699-0, pp: 1-7.

MacCartney, G.R. and T.S. Rappaport, 2017. Rural macrocell path loss models for millimeter wave wireless communications. IEEE. J. Sel. Areas Commun., 35: 1663-1677.

Maccartney, G.R., T.S. Rappaport, S. Sun and S. Deng, 2015. Indoor office wideband millimeter-wave propagation measurements and channel models at 28 and $73 \mathrm{GHz}$ for ultra-dense $5 \mathrm{G}$ wireless networks. IEEE Access, 3: 2388-2424.

Rappaport, T.S., G.R. MacCartney, M.K. Samimi and S. Sun, 2015b. Wideband millimeter-wave propagation measurements and channel models for future wireless communication system design. IEEE. Trans. Commun., 63: 3029-3056.

Rappaport, T.S., R.W. Heath, R.C. Daniels and J.N. Murdock, 2015a. Millimeter Wave Wireless Communications. Prentice Hall, Upper Saddle River, New Jersey, USA., Pages: 600.

Rappaport, T.S., S. Sun, R. Mayzus, H. Zhao and Y. Azar et al., 2013. Millimeter wave mobile communications for $5 \mathrm{G}$ cellular: It will work! . IEEE. Access, 1: 335-349.

Rappaport, T.S., Y. Xing, G.R. MacCartney Jr, A.F. Molisch, E. Mellios and J. Zhang, 2017. Overview of millimeter wave communications for 5th-generation (5G) wireless networks-with a focus on propagation models. IEEE. Trans. Antennas Propag., 65: 6213-6230.
Roh, W., J.Y. Seol, J. Park, B. Lee and J. Lee et al., 2014. Millimeter-wave beamforming as an enabling technology for $5 \mathrm{G}$ cellular communications: Theoretical feasibility and prototype results. IEEE. Commun. Mag., 52: 106-113.

Sakaguchi, K., G.K. Tran, H. Shimodaira, S. Nanba and T. Sakurai et al., 2015. Millimeter-wave evolution for $5 \mathrm{G}$ cellular networks. IEICE. Trans. Commun., 98: 388-402.

Sun, S. and T.S. Rappaport, 2017. Millimeter wave MIMO channel estimation based on adaptive compressed sensing. Proceedings of the 2017 IEEE International Conference on Communications Workshops (ICC Workshops), May 21-25, 2017, IEEE, Paris, France, ISBN:978-1-5090-1526-9, pp: 47-53.

Sun, S., T.S. Rappaport, S. Rangan, T.A. Thomas and A. Ghosh et al., 2016. Propagation path loss models for 5G urban micro-and macro-cellular scenarios. Proceedings of the 2016 IEEE 83rd International Conference on Vehicular Technology (VTC Spring), May 15-18, 2016, IEEE, Nanjing, China, ISBN:978-1-5090-1699-0, pp: 1-6.

Thomas, T.A., M. Rybakowski, S. Sun, T.S. Rappaport and H. Nguyen et al., 2016. A prediction study of path loss models from $2-73.5 \mathrm{GHz}$ in an urban-macro environment. Proceedings of the 2016 IEEE 83rd International Conference on Vehicular Technology (VTC Spring), May 15-18, 2016, IEEE, Nanjing, China, ISBN:978-1-5090-1699-0, pp: 1-5.

Tikhomirov, A., E. Omelyanchuk and A. Semenova, 2018. Recommended 5G frequency bands evaluation. Proceedings of the 2018 International Conference on Systems of Signals Generating and Processing in the Field of on Board Communications, March 14-15, 2018, IEEE,Moscow, Russia,ISBN:978-1-5386-5623-5, pp: 1-5. 